\title{
AD HOC CONSULTANTS - 2019
}

Adriana Benevides Soares - Universidade do Estado do Rio de Janeiro, Rio de Janeiro-RJ

Adriana Martins Saur - Universidade de São Paulo, Ribeirão Preto-SP

Alessandra Turini Bolsoni-Silva - Universidade Estadual Paulista Julio de Mesquita Filho, Bauru-SP

Amalia Raquel Pérez-Nebra - Centro Universitário de Brasília, Brasília-DF

Amanda Helena Rodrigues Franco - Universidade de Aveiro, Aveiro-Portugal

Ana Maria Nunes El Achkar - Universidade Salgado de Oliveira, Niteroi - RJ

André Luiz Monezi Andrade - Pontifícia Universidade Católica de Campinas, Campinas-SP

Angela Helena Marin - Universidade Federal do Rio Grande do Sul, Porto Alegre-RS

Antonio Paulo Angelico - Universidade Federal de São João del-Rei, São João del-Rei-MG

Carlos Daniel Moreira de Barros - Universidade de Lisboa, Lisboa-Portugal

Carlos Eduardo Martins Torcato - Universidade do Estado do Rio Grande do Norte, Mossoró-RN

Clarissa Mendonça Corradi-Webster - Universidade de São Paulo, Ribeirão Preto-SP

Cristian Zanon - Universidade Federal do Rio Grande do Sul, Porto Alegre-RS

Cynthia Cassoni - Universidade do Estado do Rio de Janeiro, Rio de Janeiro-RJ

Cynthia de Freitas Melo - Universidade de Fortaleza, Fortaleza-CE

Daniel Bartholomeu - Centro Universitário FIEO, Osasco-SP

Débora Dalbosco Dell'Aglio - Universidade Federal do Rio Grande do Sul, Porto Alegre-RS

Denise Falcke - Universidade do Vale do Rio dos Sinos, São Leopoldo-RS

Edna Maria Marturano - Universidade de São Paulo, Ribeirão Preto-SP

Eduardo Name Risk - Universidade Federal de São Carlos, São Carlos-SP

Elisa Kern de Castro - Universidade do Vale do Rio dos Sinos, São Leopoldo-RS

Fabiana Maris Versuti-Stoque - Universidade de São Paulo, Ribeirão Preto-SP

Fabiana Queiroga - Centro Universitário de Brasilia, Brasília-DF

Fábio Scorsolini-Comin - Universidade de São Paulo, Ribeirão Preto-SP

Felipe Valentini - Universidade São Francisco, Campinas-SP

Fernanda Aguiar Pizeta - Universidade Paulista, Ribeirão Preto-SP

Fernanda Pessolo Rocha - Centro Universitário Barão de Mauá, Ribeirão Preto-SP 
Gimol Benzaquen Perosa - Universidade Estadual Paulista Júlio de Mesquita Filho, Botucatu-SP

Karen Cristina Alves Lamas - Universidade Salgado de Oliveira, Juiz de Fora-MG

Katya Luciane Oliveira - Universidade Estadual de Londrina, Londrina-PR

Larissa Horta Esper - Universidade de São Paulo, Ribeirão Preto-SP

Leonardo Martins - Universidade Salgado de Oliveira, Niterói-RJ

Lucas Cordeiro Freitas - Universidade Federal de São João del-Rei, São João del-Rei-MG

Luciana Carla dos Santos Elias - Universidade de São Paulo, Ribeirão Preto-SP

Luciana Mourão Cerqueira e Silva - Universidade Salgado de Oliveira, Niteroi-RJ

Luciane da Rosa Piccolo - Universidade Federal do Rio Grande do Sul, Porto Alegre-RS

Manoel Antônio dos Santos - Universidade de São Paulo, Ribeirão Preto-SP

Márcia Maria Peruzzi Elia da Mota - Universidade do Estado do Rio de Janeiro, Rio de Janeiro-RJ

Maria Aparecida Crepaldi - Universidade Federal de Santa Catarina, Florianópolis-SC

Marta Regina Gonçalves Correia Zanini - Centro Universitario das Faculdades Associadas de Ensino, São João da Boa Vista-SP

Maurício Fiore - Centro Brasileiro de Análise e Planejamento, São Paulo-SP

Michele Poletto - Faculdade Ftec/IBGEN, Porto Alegre-RS

Nadia Prazeres Pinheiro Carozzo - Universidade Federal do Maranhão, São Luís-MA

Natália Martins Dias - Universidade Federal de Santa Catarina, Florianópolis-SC

Nelson Hauck Filho - Universidade São Francisco, Itatiba-SP

Paloma Pegolo de Albuquerque - Universidade Federal do Triângulo Mineiro, Uberaba-MG

Patricia Alvarenga - Universidade Federal da Bahia, Salvador-BA

Patricia Lorena Quiterio - Universidade do Estado do Rio de Janeiro, Rio de Janeiro-RJ

Rebeca Veras de Andrade Vieira - Universidade do Vale do Rio dos Sinos, São Leopoldo-RS

Renata Silva de Carvalho Chinelato - Universidade Federal de Santa Catarina, Florianópolis-SC

Rita Karina Nobre Sampaio - Universidade Estadual de Campinas, Campinas-SP

Rodrigo Rezende Ferreira - Universidade de Brasília, Brasília-DF

Rosa de Jesus Ferreira Novo - Universidade de Lisboa, Lisboa-Portugal

Sonia Regina Pasian - Universidade de São Paulo, Ribeirão Preto-SP

Sônia Maria Guedes Gondim - Universidade Federal da Bahia, Salvador-BA 
Susana Maria Gonçalves Coimbra - Universidade do Porto, Porto-Portugal

Vanessa Barbosa Romera Leme - Universidade do Estado do Rio de Janeiro, Rio de Janeiro-RJ

Vinícius Renato Thomé Ferreira - Faculdade Meridional, Passo Fundo-RS

Wanderlei Abadio de Oliveira - Universidade de São Paulo, Ribeirão Preto-SP

William Sorensen - University of Texas at Tyler, Texas-USA 